\title{
Improvement of endometrial biopsy over transvaginal ultrasound alone for endometrial surveillance in women with Lynch syndrome
}

\author{
Lotte H. M. Gerritzen • Nicoline Hoogerbrugge $\cdot$ Angèle L. M. Oei • \\ Fokko M. Nagengast - Maaike A. P. C. van Ham - Leon F. A. G. Massuger • \\ Joanne A. de Hullu
}

Published online: 6 June 2009

(c) The Author(s) 2009. This article is published with open access at Springerlink.com

\begin{abstract}
In women with hereditary non polyposis colorectal carcinoma (HNPCC) an annual gynaecological surveillance has been recommended because of an increased lifetime risk of developing endometrial and ovarian carcinoma. The aim of this study was to assess the efficacy of gynaecological surveillance with regard to endometrial and ovarian carcinoma. Included were women from families that fulfilled the revised Amsterdam criteria for HNPCC or who showed a proven mutation in one of the mismatch repair genes. An annual gynaecological surveillance was performed (transvaginal ultrasound (TVU) and CA 125 assessment). From January 2006 on, routine endometrial sampling was included. In a total number of 100 women 285 surveillance visits were performed. Among these, in 64 visits routine endometrial samplings were performed: three atypical hyperplasias and one endometrial carcinoma were diagnosed. This was significantly more than the atypical hyperplasia and two endometrial carcinomas that were detected after 28 samples performed because of abnormal
\end{abstract}

L. H. M. Gerritzen $(\bowtie)$ · A. L. M. Oei - M. A. P. C. van Ham ·

L. F. A. G. Massuger - J. A. de Hullu

Department of Obstetrics and Gynaecologic Oncology, Radboud

University Nijmegen Medical Centre, P.O. Box 9101, 6500 HB

Nijmegen, The Netherlands

e-mail: lotteg50@hotmail.com

N. Hoogerbrugge

Department of Medical Oncology, Radboud University Medical

Centre Nijmegen, P.O. Box 9101, 6500 HB Nijmegen,

The Netherlands

F. M. Nagengast

Department of Gastroenterology, Radboud University Nijmegen

Medical Centre, P.O. Box 9101, 6500 HB Nijmegen,

The Netherlands surveillance results in 221 visits. There were no interval carcinomas. One invasive ovarian carcinoma stage IIIC was diagnosed at ovarian surveillance. Endometrial surveillance with routine endometrial sampling in women with HNPCC is more efficient in diagnosing endometrial (pre)malignancies than TVU only. Ovarian surveillance is not capable of diagnosing early stage ovarian carcinoma. Prophylactic hysterectomy in HNPCC should be restricted to women in whom abdominal surgery for other reasons is performed and to those with particularly increased risk such as MSH6 mutation carriers and/or women with multiple relatives with endometrial carcinoma.

Keywords Endometrial carcinoma $\cdot$ Endometrial sampling $\cdot$ HNPCC $\cdot$ Ovarian carcinoma $\cdot$ Screening . Surveillance

$\begin{array}{ll}\text { Abbreviations } \\ \text { HNPCC } & \begin{array}{l}\text { Hereditary non polyposis colorectal } \\ \text { carcinoma }\end{array} \\ \text { TVU } & \begin{array}{l}\text { Transvaginal ultrasound } \\ \text { Mismatch repair gene }\end{array} \\ \text { BSO gene } & \begin{array}{l}\text { Bilateral salpingo-oophorectomy } \\ \text { LNG-IUS }\end{array} \\ \begin{array}{l}\text { Levonorgestrel releasing intrauterine } \\ \text { system }\end{array} \\ \text { CA 125 } & \text { Cancer antigen 125 } \\ \text { BRCA gene } & \text { Breast cancer gene }\end{array}$

Introduction

Lynch syndrome is an autosomal dominantly inherited disorder characterized by a marked increase in carcinoma susceptibility caused by a germline mutation in one of the mismatch repair (MMR) genes, which are $M L H 1, M S H 2$, 
MSH6 and PMS2 [1, 2]. Hereditary non polyposis colorectal carcinoma (HNPCC) is defined as positive revised Amsterdam Criteria [3] and/or a proved mutation in one of the MMR genes. Next to an enhanced lifetime risk for colorectal carcinoma (18-73\%), women with HNPCC have an increased lifetime risk to develop endometrial and ovarian carcinoma, being $27-70 \%$ and 3-28\%, respectively [4, 5]. $M S H 2$ mutation carriers seem to be at higher risk for endometrial carcinoma than $M L H 1$ carriers, and $M S H 6$ carriers may be at even greater risk up to $70 \%[6,7]$.

For women with HNPCC current surveillance protocols recommend annual transvaginal ultrasound (TVU) and determination of tumor markers such as CA 125 [2, 8]. Whether either endometrial surveillance or prophylactic hysterectomy is optimal in terms of benefits and risks is still subject of debate. This gives major implications for risk management counselling. Good quantification of the diagnostic accuracy of endometrial surveillance in daily practice is needed, as prophylactic surgery is irreversible.

There is conflicting evidence as far as the efficacy of endometrial surveillance with TVU in women with HNPCC is concerned. One study found three pre-malignancies and one interval endometrial carcinoma [2]. Another study failed to demonstrate any clinical benefit of the surveillance with TVU and also found two interval endometrial carcinomas, diagnosed at an early stage [8].

The lack of evidence of endometrial surveillance with TVU combined with the favourable prognosis for endometrial carcinoma patients, questioned the need for surveillance. However, in a recent study by Renkonen et al. endometrial carcinoma surveillance appeared to be highly effective with the addition of endometrial sampling. Moreover, the tumors that were identified at routine endometrial sampling were smaller and tended to be at an earlier stage compared to the symptomatic cases [9].

A large retrospective study in women with Lynch syndrome suggested that prophylactic hysterectomy and bilateral salpingo-oophorectomy (BSO) might be effective strategies. Serious complications such as postoperative infection or bleeding do occur but are rare [4, 10]. It might be suggested that after hysterectomy colonoscopy may be more painful compared to women who did not have a hysterectomy [11]. Considering the mean life time risk of $6-8 \%$ on ovarian carcinoma in women with HNPCC there is neither evidence nor consensus as to whether this risk is high enough to perform prophylactic surgery.

The aim of the present study was to evaluate the efficacy of annual gynaecological surveillance in women with HNPCC with regard to endometrial and ovarian carcinoma at the Family Cancer Clinic at the tertiary referral centre of the Radboud University Nijmegen Medical Centre, The Netherlands.

\section{Materials and methods}

Study population

All female members with either colorectal carcinoma (or another HNPCC related carcinoma) or who are first degree member of a cancer patient in families with HNPCC, who visited the Family Cancer Clinic of the Radboud University Nijmegen Medical Centre with the uterus (and/or adnexa) in situ were referred for annual gynaecological surveillance to the department of gynaecological oncology. The Family Cancer Clinic is a multidisciplinary team of clinicians, including a clinical geneticist, a gastroenterologist, a surgeon, a medical oncologist and a gynaecological oncologist with weekly meetings where all different aspects of the medical policy of patients with HNPCC are discussed.

Data used for this analysis were collected from January 1997 till February 2008. Primary surveillance was started from the age of 30 or 5 years before the first family member was diagnosed with endometrial or ovarian carcinoma. Women under the age of 30 were offered one single surveillance visit with general information to return for annual surveillance after their 30th anniversary. When during the surveillance program a mutation was found in a family all included family members who appeared to be no carrier of the mutation were excluded from the surveillance program.

At primary surveillance information on age of menarche, use of oral contraceptives, parity, medical history, occurrence of cancer in the family, mutation test results and the pre-and post-menopausal status were collected. All patients with abnormal findings at surveillance and/or other important issues (such as prophylactic surgery) were discussed at the weekly meeting.

\section{Surveillance}

Surveillance visits were performed annually and consisted of pelvic examination, TVU and measurement of serum CA 125 levels. Women were asked to report clinical symptoms. Before 2006 endometrial sampling (micro curettage in the outpatient clinic) was only indicated in case of postmenopausal/irregular bleeding and/or abnormal findings at TVU: in postmenopausal women when the TVU showed irregularity, polyps or endometrial thickness more than $4 \mathrm{~mm}$ and in premenopausal women when there was an endometrial thickness of more than $12 \mathrm{~mm}$ preovulatory, an irregularity/polyp or when the ultrasound was not well assessable. In January 2006 routine endometrial sampling was added to the surveillance. When microcurettage was technically impossible or when insufficient material was obtained, hysteroscopy and/or curettage were 
performed to obtain representative endometrial tissue. In case of a (pre)malignancy of the uterus hysterectomy and BSO was advised. The cut-off value for CA 125 was $35 \mathrm{U} / \mathrm{ml}$. With respect to the ovaries, in case of an abnormal pelvic examination and/or TVU and/or CA 125 , revision after 3 months was advised, unless findings were highly suspicious for a malignancy in which case the patient was asked to return earlier for follow-up or diagnostic surgery such as laparoscopy.

\section{Prophylactic surgery}

At primary counselling patients were informed on the advantage (possible earlier diagnosis of (pre)malignancy) and disadvantages of surveillance (no prevention of carcinoma and the risk of false positive findings resulting in a higher rate of diagnostic surgery). Patients were informed that prophylactic surgery was not the standard of care in our institute, unless the patient needed abdominal surgery for other reasons e.g. colorectal carcinoma. In case the patient nevertheless opted for prophylactic surgery this was discussed in the multidisciplinary team of the family cancer clinic.

In case of surgery for prophylactic or therapeutic reasons, all removed specimens such as uterus, ovaries and tubes were separately examined by an experienced gynaecologic pathologist. After hysterectomy and BSO women were dismissed from further surveillance. In case of surgery with saving the uterus and/or one or two ovaries, patients returned to the surveillance program.

\section{Results}

\section{Patient characteristics}

A total number of 100 women fulfilled the inclusion criteria from January 1997 till February 2008 and were enrolled in our surveillance program.

The median age of the women was 46 years (range 2372 years). In 12 women the mutation status was not (yet) known because they were in the procedure of testing $(n=5)$ or the patient refused DNA analysis $(n=7)$. Five patients were tested negative for the MMR-gene mutation known to be present in their family and were dismissed from further surveillance. In another 16 patients (and their families) DNA analysis did not reveal a mutation; however, they preferred surveillance because the family fulfils the revised Amsterdam criteria [3]. An overview of the patient characteristics is given in Table 1. Six women underwent prophylactic surgery: one BSO (with a hysterectomy in the past) because the patient preferred BSO above surveillance and discontinued surveillance afterwards; two patients
Table 1 Patient characteristics of study population (100 women) at primary surveillance

\begin{tabular}{ll}
\hline Median age & 46 years (range 23-72 years) \\
Median follow-up & 1 year (range 0-16 years) \\
Median number of visits & 1 (range 1-16) \\
\hline & Number of patients \\
\hline Type mutation & 22 \\
MLH1 & 22 \\
MSH2 & 23 \\
MSH6 & 16 \\
No mutation found & 5 \\
Tested, no results yet or unknown & 5 \\
Tested negative during & \\
$\quad$ surveillance & \\
(family positive) & \\
Unknown (not tested) & 7 \\
Menopausal state & \\
Premenopausal & 72 \\
Postmenopausal & 22 \\
Unknown & 6 \\
\hline
\end{tabular}

opted nevertheless for prophylactic hysterectomy with BSO and three women developed colorectal carcinoma and preferred after counselling prophylactic hysterectomy and BSO in the same operation session.

\section{Endometrial surveillance}

A total number of 100 women underwent 285 surveillance visits till February 2008. In seven patients hysterectomy and BSO was performed due to endometrial (pre)malignancies. Since January 200649 routine endometrial samplings and 15 hysteroscopies and/or curettages in 64 surveillance visits diagnosed three patients with atypical hyperplasia and one patient with endometrial carcinoma (endometrial carcinoma stage IB in patient with $M S H 6$ mutation; curettage showed atypical hyperplasia while definitive histopathology showed invasive disease). The (pre)malignancies were found in $6.3 \%$ of the visits which was significantly higher $(P=0.026)$ compared to the period of 8 years before the introduction of routine endometrial sampling: 17 microcurettages and 15 hysteroscopies and/or curettages had been performed because of abnormal TVU and/or complaints in 221 surveillance visits. The three (pre)malignities found concerned $1.4 \%$ of the surveillance visits. One patient was diagnosed with atypical hyperplasia and two patients with endometrial carcinomas. One endometrial carcinoma patient with $M S H 2$ mutation had irregular bleeding at the first visit, which appeared to be stage IIIC disease (prevalent case) and the other patient with MLH1 mutation had an abnormal endometrial 


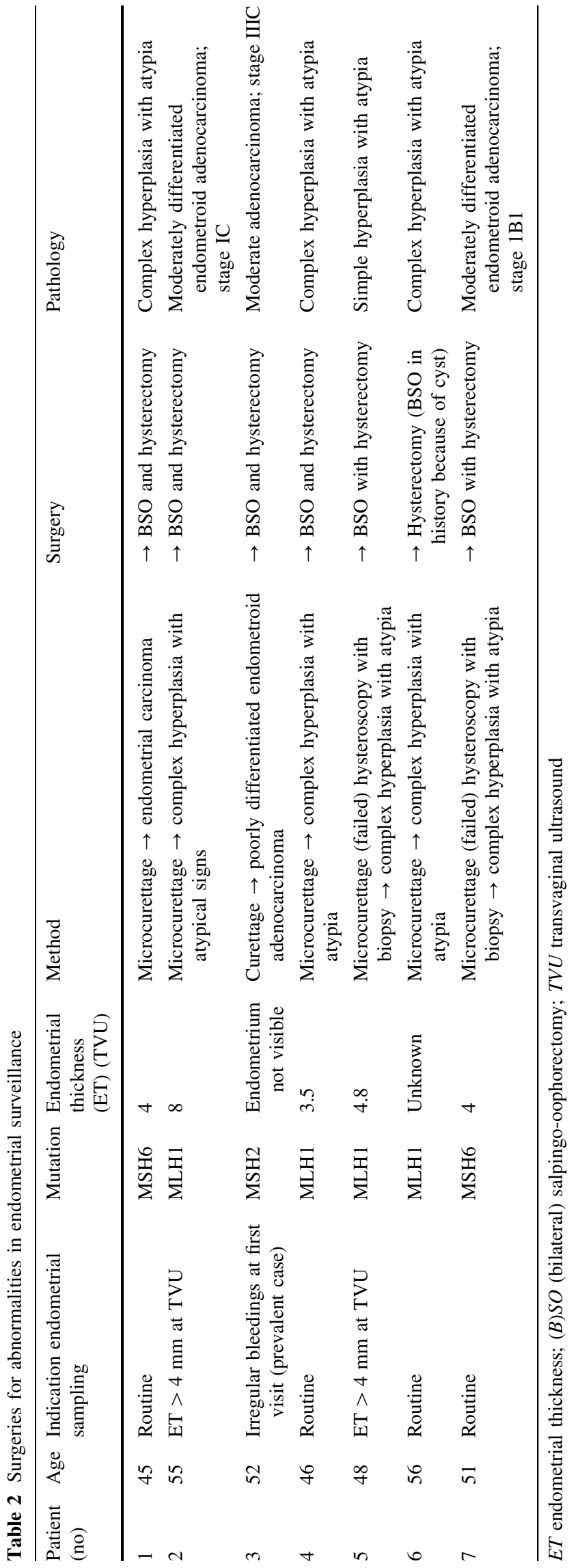

thickness of $8 \mathrm{~mm}$ at her third surveillance visit. There were no interval carcinomas. See Table 2 for an overview of the seven patients who underwent surgery for their endometrial (pre)malignancies (four hyperplasia with atypia and three endometrial carcinomas).

Ovarian surveillance

In 100 patients, 12 interval surveillance visits and three CT scans were performed due to suspicious ovaries with TVU or raise of serum CA 125. Eight surgeries were performed: one hysterectomy with BSO, two BSO, one SO, three laparoscopic explorations and one abdominal exploration with debulking. The final pathological result showed normal ovaries in five patients, one borderline malignancy and one mature teratoma; one patient with $\mathrm{MSH} 2$ mutation appeared to have stage IIIC ovarian carcinoma. For an overview of these patients see Table 3 .

\section{Surveillance results}

The median number of surveillance visits per patient was 1 (range 1-16) and the median follow-up was 1 year (range $0-15)$ in a total of 286 women-years. The average followup was 2.8 years (See Table 1). Table 4 gives an overview of the results of the different surveillance tools.

At the time of this analysis, 54 women still participate in the surveillance program. Of the other 46 women, 17 women had undergone hysterectomy with BSO (six because of prophylactic reasons or because of colorectal carcinoma surgery), seven women underwent surveillance somewhere else, 12 women were lost for follow-up, five patients appeared to be a non carrier, four patients were advised to start screening after their 30th birthday and one patient (temporary) stopped surveillance on their own initiative. During surveillance one patient died from ovarian carcinoma and two women died from colorectal carcinoma. No women died from endometrial carcinoma. The patients with endometrial carcinoma were, respectively, 8, 11 and 21 months after their diagnosis and did not show any sign of recurrence.

\section{Discussion}

In women with HNPCC asymptomatic endometrial (pre)malignancies can be identified by annual endometrial surveillance, which preferably includes routine endometrial sampling than TVU alone. Based on these results, combined with data from literature, we conclude that endometrial carcinoma surveillance in women with HNPCC could be effective and therefore the medical indications for prophylactic hysterectomy should be restricted [9]. 


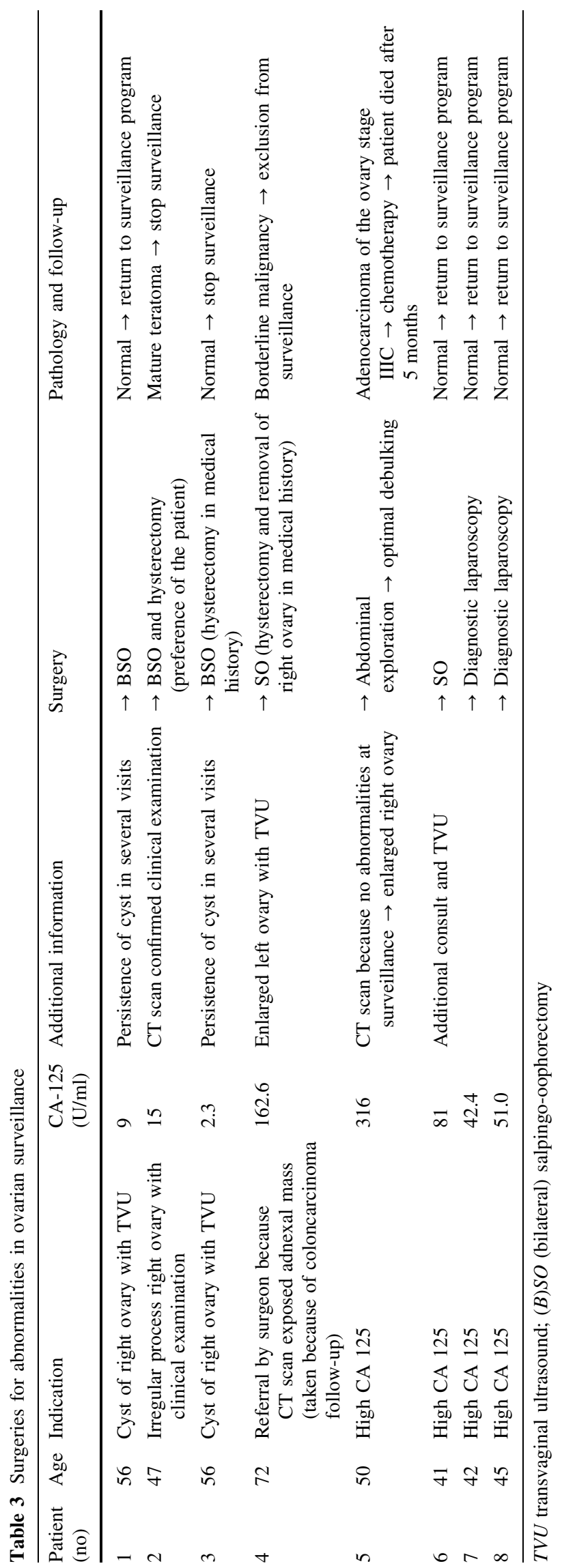

In our study we identified in total seven patients with endometrial (pre)malignancies: adding of routine endometrial sampling led to the diagnosis endometrial (pre)malignancy in four of these cases, which is in $6.3 \%$ of 64 visits. The other (pre)malignancies were found in 221 visits where specimens were collected because of abnormal surveillance results $(1.4 \%)$. From our data there is no good correlation between endometrial thickness and (pre)malignant pathology. This emphasizes that it is unknown whether endometrial carcinoma in women with HNPCC always arises through the (pre)malignant precursor hyperplasia. Our results are in concordance with the findings by Renkonen et al. who analysed the role of routine endometrial sampling and showed an increase in finding (pre)malignancies at an earlier stage, like we did. Endometrial sampling can be performed without sedation at the outpatient clinic and has similar sensitivity as dilatation and curettage in detecting endometrial abnormalities [9, 12, 13]. We did not find any interval carcinoma, possibly due to a limited follow-up which is a defect in our study.

Currently results of surveillance may be improved by a better selection of women at risk, e.g. by selecting only MMR-gene mutation carriers. A substantial part of our patients were members of HNPCC families without an established mutation. Microsatellite instability (MSI) of tumor DNA is a hallmark of the presence of MMR dysfunction. Currently MSI investigation of the tumor is a helpful tool to identify families that are prone to a MMR gene mutation. When MSI analysis in the most suspected carcinoma of the family turns out to be MSI-negative the diagnosis of Lynch syndrome is unlikely and in most cases such families will be excluded from further endometrial surveillance [14].

Prophylactic surgery can be an option for prevention of gynaecological malignancies in women with HNPCC. A study by Schmeler et al. provides strong evidence that uterine and ovarian carcinomas can be prevented in women with Lynch syndrome by prophylactic surgery. They found that patients who underwent hysterectomy did not develop endometrial carcinoma, whereas patients who did not undergo hysterectomy, endometrial carcinoma did occur. Similar findings were reported for ovarian carcinoma and BSO in patients with Lynch syndrome [4, 15]. The removal of the uterus and adnexa (by vaginal, abdominal or laparoscopic procedure) may cause infection, bleeding, ureter/ bladder and bowel injuries, but these complications are rare [16]. After hysterectomy, colonoscopy may be more difficult and painful and is associated with a reduction of polyp detection rate [11]. Another issue is the good prognosis in case endometrial carcinoma is detected, because it can mostly be treated with hysterectomy and BSO. At the moment the 5-years survival for women with HNPCC with endometrial carcinoma is more than $80 \%$ [17]. It is 
Table 4 Results of surveillance tools and interventions

\begin{tabular}{|c|c|c|c|c|}
\hline \multirow{2}{*}{$\begin{array}{l}\text { Surveillance tool or } \\
\text { diagnostic intervention } \\
\text { TVU }\end{array}$} & \multicolumn{2}{|c|}{$\begin{array}{l}\text { Number of } \\
\text { patients }\end{array}$} & \multicolumn{2}{|l|}{ Outcome } \\
\hline & 285 & 240 & Normal & \\
\hline & & 23 & Ovarian abnormality $\rightarrow$ cyst & 12 additional consults $\rightarrow 8$ surgeries: \\
\hline & & & & $1 \rightarrow$ ovarian carcinoma $^{a}$ \\
\hline & & & & $1 \rightarrow$ mature teratoma \\
\hline & & & & $1 \rightarrow$ borderline malignancy \\
\hline & & & & $5 \rightarrow$ normal ovaries \\
\hline & & 22 & Endometrial abnormality & $\rightarrow$ Microcurettage/hysteroscopy with biopsy \\
\hline & & & $12 \rightarrow$ endometrial thickness increased & or curettage \\
\hline & & & $5 \rightarrow$ polyp & \\
\hline & & & $5 \rightarrow$ myoma & \\
\hline \multirow[t]{6}{*}{ CA $125(\mathrm{U} / \mathrm{ml})$} & 270 & 265 & $\leq 35$ & \\
\hline & & 5 & $>35$ & $\begin{array}{l}\rightarrow 316 \rightarrow \text { also enlarged ovary } \rightarrow \text { ovarian } \\
\text { carcinoma }^{\mathrm{a}}\end{array}$ \\
\hline & & & & $\begin{array}{l}\rightarrow 162,6 \rightarrow \text { also enlarged ovary } \rightarrow \text { exploration: } \\
\text { borderline malignancy }\end{array}$ \\
\hline & & & & $\begin{array}{l}\rightarrow 81 \rightarrow \text { repeat of measurement: same } \\
\quad \text { outcome } \rightarrow \text { exploration } \rightarrow \text { normal anatomy }\end{array}$ \\
\hline & & & & $\rightarrow 42,4 \rightarrow$ exploration $\rightarrow$ normal anatomy \\
\hline & & & & $\rightarrow 51,0 \rightarrow$ exploration $\rightarrow$ normal anatomy \\
\hline \multirow{4}{*}{$\begin{array}{l}\text { Microcurettage/curettage/biopsy } \\
\text { taken as routine (after January } \\
2006 \text { ) }\end{array}$} & 64 & 49 & Normal & \\
\hline & & 11 & Inadequate material & Further analysis as possible ${ }^{\mathrm{b}}$ \\
\hline & & 4 & Abnormal & $3 \rightarrow$ hyperplasia with atypia \\
\hline & & & & $1 \rightarrow$ endometrial carcinoma \\
\hline \multirow{4}{*}{$\begin{array}{l}\text { Microcurettage/curettage/biopsy } \\
\text { taken after complaints } \\
\text { or abnormal TVU (before } \\
\text { Jan 2006) }\end{array}$} & 32 & 24 & Normal & \\
\hline & & 5 & Inadequate material & Further analysis as possible ${ }^{\mathrm{b}}$ \\
\hline & & 3 & Abnormal & $1 \rightarrow$ hyperplasia with atypia \\
\hline & & & & $2 \rightarrow$ endometrial carcinoma \\
\hline
\end{tabular}

${ }^{a}$ Same patient with ovarian cancer: enlarged ovary and elevated CA 125

b Return to surveillance for in total two patients without final pathological diagnosis, but low clinical suspicion

unlikely we could achieve a better prognosis by a surveillance program.

In the future, another alternative for patients with endometrial hyperplasia or early endometrial carcinoma could be a levonorgestrel releasing intrauterine system (LNG-IUS) with frequent endometrial sampling. A recent promising small study has shown that LNG-IUS can provide reduction of endometrial hyperplasia and early stage endometrial carcinoma; however, the evidence is limited [18]. An international study on the topic has started in the UK.

In the general population there is evidence that oral contraceptives decrease the incidence of both ovarian and endometrial carcinoma. Oral contraceptives might also decrease carcinoma risk in patients with Lynch syndrome, but there is lack of data in these women [19].

Regarding the risk of ovarian carcinoma in HNPCC mutation carriers, surveillance of the ovaries is disputable. Nevertheless, we included surveillance of the ovaries in our program. In our study one patient without complaints was diagnosed with advanced ovarian carcinoma based on TVU and CA 125 and she died within 5 months after diagnosis. Surveillance for ovarian carcinoma in BRCA mutations carriers has proven to be inefficient, while these women have an even higher lifetime risk for developing ovarian carcinoma as compared to women with HNPCC [20]. Considering the mean lifetime risk of $6-8 \%$ on ovarian carcinoma in women with HNPCC there is neither evidence nor consensus as to whether this risk is high enough to perform prophylactic surgery.

Hysterectomy with BSO is discussed with a HNPCC patients who will undergo abdominal surgery for other reasons e.g. colorectal carcinoma as well as in women with an MSH6 mutation and/or multiple relatives with endometrial carcinoma. For decision making in the treatment of women with HNPCC with respect to prophylactic surgery we wanted to point out the necessity of multidisciplinary 
teams of clinicians, including a clinical geneticist, a gastroenterologist, a surgeon, a medical oncologist and a gynaecological oncologist. It is necessary to be informed by the gastroenterologist whether an optimal colonoscopy is possible when a hysterectomy is planned and vice versa. This will also provide the possibility of combined surgeries between surgical and gynaecological specialties, reducing morbidity in patients [21].

In conclusion, gynaecological surveillance with TVU and CA 125 is efficient for endometrial carcinoma (and will even be improved by routine endometrial sampling) but not for ovarian carcinoma. A multidisciplinary setting will further optimise the care and decision making in women with HNPCC. In women with HNPCC we recommend further studies to investigate the prevention of endometrial carcinoma by levonorgestrel releasing intra uterine system and the role of prophylactic surgery.

Open Access This article is distributed under the terms of the Creative Commons Attribution Noncommercial License which permits any noncommercial use, distribution, and reproduction in any medium, provided the original author(s) and source are credited.

\section{References}

1. Lynch HT, Shaw MW, Magnuson CW et al (1966) Hereditary factors in cancer. Study of two large midwestern kindreds. Arch Intern Med 117:206-212. doi:10.1001/archinte.117.2.206

2. Rijcken FE, Mourits MJ, Kleibeuker JH et al (2003) Gynecologic screening in hereditary nonpolyposis colorectal cancer. Gynecol Oncol 91:74-80. doi:10.1016/S0090-8258(03)00371-8

3. Vasen HF, Watson P, Mecklin JP et al (1999) New clinical criteria for hereditary nonpolyposis colorectal cancer (HNPCC, Lynch syndrome) proposed by the international collaborative group on HNPCC. Gastroenterology 116:453-456. doi:10.1016/ S0016-5085(99)70510-X

4. Schmeler KM, Lynch HT, Chen LM et al (2003) Prophylactic surgery to reduce the risk of gynecologic cancers in the Lynch syndrome. N Engl J Med 354:261-269. doi:10.1056/NEJMoa 052627

5. Oei AL, Massuger LF, Bulten J et al (2006) Surveillance of women at high risk for hereditary ovarian cancer is inefficient. $\mathrm{Br}$ J Cancer 94:814-819. doi:10.1038/sj.bjc.6603015

6. Vasen HF, Stormorken A, Menko FH et al (2001) MSH2 mutation carriers are at higher risk of cancer than MLH1 mutation carriers: a study of hereditary nonpolyposis colorectal cancer families. J Clin Oncol 19:4074-4080
7. Wijnen J, de Leeuw W, Vasen H et al (1999) Familial endometrial cancer in female carriers of MSH6 germline mutations. Nat Genet 23:142-144. doi:10.1038/13773

8. Dove-Edwin I, Boks D, Goff S et al (2002) The outcome of endometrial carcinoma surveillance by ultrasound scan in women at risk of hereditary nonpolyposis colorectal carcinoma and familial colorectal carcinoma. Cancer 94:1708-1712. doi:10.1002/ cncr. 10380

9. Renkonen-Sinisalo L, Bützow R, Leminen A et al (2007) Surveillance for endometrial cancer in hereditary nonpolyposis colorectal cancer syndrome. Int J Cancer 120:821-824. doi:10.1002/ ijc. 22446

10. Pistorius S, Kruger S, Hohl R et al (2006) Occult endometrial cancer and decision making for prophylactic hysterectomy in hereditary nonpolyposis colorectal cancer patients. Gynecol Oncol 102:189-194. doi:10.1016/j.ygyno.2005.12.032

11. Adams C, Cardwell C, Cook C et al (2003) Effect of hysterectomy status on polyp detection rates at screening flexible sigmoidoscopy. Gastrointest Endosc 57:848-853. doi:10.1016/S0016-5107(03) 70019-4

12. Lindor NM, Petersen GM, Hadley DW et al (2006) Recommendations for the care of individuals with an inherited predisposition to Lynch Syndrome. JAMA 296:1507-1517. doi:10.1001/jama. 296.12.1507

13. Clark TJ, Mann CH, Shah N et al (2002) Accuracy of outpatient endometrial biopsy in the diagnosis of endometrial cancer: a systematic quantitative review. Br J Obstet Gynaecol 109:313-321

14. Overbeek LI, Kets CM, Hebeda KM et al (2007) Patients with an unexplained microsatellite instable tumour have a low risk of familial cancer. Br J Cancer 96:1605-1612. doi:10.1038/sj.bjc. 6603754

15. Chen LM, Yang KY, Little SE et al (2007) Gynecologic cancer prevention in Lynch syndrome/hereditary nonpolyposis colorectal cancer families. Obstet Gynecol 110:18-25

16. Makinen J, Johansson J, Tomas C et al (2001) Morbidity of 10 110 hysterectomies by type of approach. Hum Reprod 16:14731478. doi:10.1093/humrep/16.7.1473

17. Vasen HF, Watson P, Mecklin JP et al (1994) The epidemiology of endometrial cancer in hereditary nonpolyposis colorectal cancer. Anticancer Res 14:1675-1678

18. Varma R, Sinha D, Gupta J (2006) Non-contraceptive uses of levonorgestrel-releasing hormone system (LNG-IUS) — a systematic enquiry, overview. Eur J Obstet Gynecol Reprod Biol 125:928. doi:10.1016/j.ejogrb.2005.10.029

19. The ESHRE Capri Workshop Group (2005) Noncontraceptive health benefits of combined oral contraception. Hum Reprod Update 11:513-525. doi:10.1093/humupd/dmi019

20. Hermsen BB, Olivier RI, Verheijen RH et al (2007) No efficacy of annual gynaecological screening in BRCA1/2 mutation carriers; an observational follow-up study. Br J Cancer 96:1335-1342

21. Lu KH (2008) Hereditary gynecologic cancers: differential diagnosis, surveillance, management and surgical prophylaxis. Fam Cancer 7:53-58. doi:10.1007/s10689-007-9144-x 